\title{
Evaluation of BepanGel Hydrogel Efficacy and Tolerability Using an Abrasive Wound Model in a Within-Person, Single-Center, Randomized, Investigator-Blind Clinical Investigation
}

\author{
Lei Zhang - Raffaella de Salvo · Sonja Trapp - Walter Wigger-Alberti • \\ Ragna Williams · Lucie Delcour • Bart Rossel • Maarten T. Huisman (D)
}

Received: June 29, 2020 / Published online: August 2, 2020

(C) The Author(s) 2020

\begin{abstract}
Introduction: Over the last few years, it has been demonstrated that a moist environment enhances the healing process and reduces scar formation of wounds. Such moist conditions can be created and maintained using hydrogels. The aim of this study was to evaluate wound healing, cooling efficacy, local tolerability, and cosmetic appearance of abrasive wounds treated with BepanGel wound care hydrogel.

Methods: This study was designed as a withinperson, single-center, randomized, investigatorblind clinical investigation comparing a hydrogel-treated test field with an untreated test field in an abrasive wound model. In 33
\end{abstract}

Digital Features To view digital features for this article go to https://doi.org/10.6084/m9.figshare.12696644.

Electronic supplementary material The online version of this article (https://doi.org/10.1007/s13555020-00432-5) contains supplementary material, which is available to authorized users.

L. Zhang $\cdot$ R. de Salvo $\cdot$ S. Trapp

Bayer Consumer Care AG, Peter Merian-Strasse 84, 4002 Basel, Switzerland

W. Wigger-Alberti · R. Williams

Bioskin GmbH, Messberg 4, 20095 Hamburg,

Germany

L. Delcour · B. Rossel · M. T. Huisman ( $\square)$

Oystershell Laboratories, Nijverheidsweg 10, 9820

Merelbeke, Belgium

e-mail:mh@oystershell.be subjects, two small superficial wounds were induced on the non-dominant forearms. Wounds were treated with BepanGel and covered with a standard semi-occlusive wound plaster or covered with a plaster alone for 11 consecutive days. Wound healing efficacy, cooling effect, and tolerability of the treatment were assessed over 12 investigational days. During follow-up at day 31, the cosmetic appearance of the wounds was evaluated.

Results: On day 12, the test field treated with BepanGel was completely healed in nearly all subjects $(97.0 \%)$ in contrast with the test field treated with a plaster alone $\left(18.2 \%, \mathrm{AUC}_{\text {days } 2-12}\right.$ $p<0.0001)$ as assessed by a blinded investigator. Two-thirds of the unblinded subjects indicated an immediate cooling effect of the hydrogel $(p=0.0555)$. At the end of the investigation, the cosmetic appearance of the BepanGel-treated test fields scored superior to the fields treated with a plaster alone as evaluated by a blinded investigator $(p=0.0005)$ and the unblinded subjects $(p=0.0078)$. The hydrogel was generally well tolerated and no signs of infection or adverse events (AEs) related to the treatment were observed.

Conclusion: This evaluation shows that treatment of superficial cutaneous wounds with BepanGel results in improved wound healing as demonstrated by faster wound closure and a considerably better cosmetic appearance, while providing immediate cooling. 
Trial Registration Number: EUDAMED-No.: CIV-19-09-029744.

Keywords: Abrasive wound model; BepanGel; Cooling; Cosmetic appearance; Efficacy; Flamozil; Hydrocolloid; Hydrogel; Tolerability; Zoralan

\section{Key Summary Points}

\section{Why carry out this study?}

Superficial minor wounds are standardly treated by cleansing with tap water or saline and use of a plaster, while there is growing evidence available reporting enhanced healing of wounds and reduced scarring when healing in a moist environment.

The current clinical investigation evaluated the healing efficacy, cooling effect, and cosmetic outcome of abrasive wounds treated with a moisturizing hydrogel.

\section{What was learned from the study?}

BepanGel (Flamozil, Zoralan Wound) wound care hydrogel clearly demonstrates a superior effect over the standard treatment (plaster) as shown by higher reepithelialization rates, an improved cosmetic outcome, and a slight cooling effect in superficial abrasive wounds.

A moist wound environment increases healing efficacy and outcomes of small superficial wounds, without posing any additional risk in comparison with the standardly used plaster.

\section{INTRODUCTION}

Minor superficial acute wounds such as abrasions, lacerations, and incisions as well as superficial thermal wounds are part of our everyday life. Such wounds result in disruption of the normal anatomic epidermal structure and function. Usually, healing of superficial wounds proceeds in a complex orderly and timely reparative process that completely restores the affected tissue and function, although formation of scars might be possible [1]. Standard treatment for such typical everyday minor wounds consists of thorough cleansing with tap water or saline followed by the use of plasters that are able to provide a barrier against dirt, contamination, and external influences and as such protect the wound and improve healing. Research over the last few years demonstrates that one of the main external factors responsible for optimal wound healing is hydration [2]. Creation of a moist wound environment, e.g., with ointments, results in improved and accelerated healing, reduces pain, and probably also restricts scar formation in comparison with conventional wound treatment $[3,4]$.

Hydrogels provide such a moist environment. Hydrogels are water-based polymers designed to prevent wounds from drying out in order to stimulate wound healing, decrease granulation and subsequent scarring, prevent cracking of the wound, and form a barrier against dirt, contamination, and external influences. The beneficial effects of hydrogels have been demonstrated in several types of wounds, including necrotic and sloughy wounds, chronic skin wounds, corneal wounds, and burn wounds [5-9]. BepanGel wound care hydrogel, also known as Flamozil or Zoralan Wound, is a paraben-free, unmedicated, acidified hydrogel that forms a semi-occlusive, breathable protective film on the wound. The hydrogel is composed of a dispersion of an acidic carbomer in water, stabilized by carnosine, a basic dipeptide naturally present in the skin. It also contains a certain percentage of fatty substances which help to reduce the evaporation of water. Presence of the carbomer results in the principal mode of action of the hydrogel by ensuring optimal hydration for both dry and wet wounds. The activity of the carbomer depends on the circumstances: in dry wounds it gives off moisture to prevent cracking of the wound and formation of a scab. In wet wounds it absorbs the excess of wound exudates. In addition, the acidity of the carbomer 
polymer keeps the $\mathrm{pH}$ of the wound environment low, thus preventing (in a natural, physical way) microbial growth. BepanGel has been CE marked as a medical device and marketed since 2007. Over two million units have been put on the market.

The objective of this study was to assess the wound healing properties of BepanGel hydrogel on small everyday epidermal wounds in comparison with the standard treatment (wound plaster) in a controlled clinical setting. The primary objectives were to evaluate wound healing-based on re-epithelialization properties-and cooling properties. The secondary objective was to assess the local tolerability of the hydrogel. In addition, the cosmetic appearance of healed wounds was evaluated; lastly, the product performance and acceptance as experienced by the subjects were assessed.

In order to realize these objectives, the abrasive wound model was selected on the basis of its (1) reflection of small epidermal wounds obtained by minor accidents, (2) relevance for the evaluation of healing properties of different products for superficial wounds, (3) ability to significantly detect differences in wound healing properties of test products, and (4) standardization and clinical validation of this method [10]. In the abrasive wound model, small superficial abrasions are induced on the forearms of subjects by repeatedly scrubbing the skin with a surgical brush until the first signs of uniform glistening and punctuate bleeding are observed.

\section{METHODS}

\section{Clinical Study}

This study was designed as a within-person, single-center (bioskin Research Center Dermatology (RCD), Hamburg, Germany), randomized, investigator-blind clinical investigation. The investigational design was blinded for the investigator who performed the clinical assessments, but could not be blinded for the subjects and study nurse. Since the study was designed as a within-person investigation, subjects were aware of the treatment allocation. The study nurse who regularly treated the induced wounds needed to be informed about which wounds to treat. To keep the blinding, the investigator was not involved in treatment procedures and the study nurse was not involved in clinical assessments. The clinical investigation was conducted in accordance with local law, the Declaration of Helsinki 1964 and its later amendments, and ISO 14155:2011. Written informed consent was obtained for all included subjects. This study is registered at BfArM and EC (EUDAMED-No.: CIV-19-09029744). The study was approved by the ethics committee of the medical association in Hamburg (Ethik-Kommission der Ärztekammer Hamburg, Weidestraße 122b, 22083 Hamburg, Germany).

\section{Study Participants and Sample Size}

A total of 40 healthy volunteers (at least 18 years old, male and female) were screened with the goal of including 33 eligible subjects and expectation of at least 27 evaluable subjects, which was considered to be sufficient to meet the primary and secondary objectives of the clinical investigation. The sample size was not based on a formal sample size calculation, but defined according to the Guidance for Industry on Skin Irritation and Sensitization Testing of Generic Transdermal Drug Products as advised by the FDA [11]. According to FDA guidance 30 subjects would have been sufficient. On top of this an additional dropout rate of $10 \%$ was considered; therefore, 33 subjects were randomized.

Subjects were eligible at screening if they presented with healthy skin on the forearms, determined by a physical examination and demonstrated by the absence of disease findings unless the investigator considered an abnormality to be irrelevant to the outcome of the clinical investigation. As dark-skinned persons tend to develop hyperpigmentation after wound healing, only skin types I to III (Fitzpatrick scale) were included in the investigation. Written informed consent was obtained for all included subjects. 


\section{Abrasive Wound Model}

The standardized abrasive wound model is ideal to assess healing properties of products intended for superficial wounds and was performed as originally described by Wigger-Alberti et al. [10]. In brief, two small superficial, abrasive wounds (approximately $1.2 \mathrm{~cm}$ in diameter) were induced on the respective non-dominant forearm of each subject using a sterile surgical hand brush. Before wounding, the forearms were disinfected with a standard alcohol-based antiseptic. Epidermal abrasive wounds were induced under visual control by the same trained study nurse, who repeatedly scrubbed the skin with a sterile surgical hand brush using moderate pressure until first signs of uniform glistening and punctuate redness were observed. Thus, it could be ensured that the wounds only reached the uppermost dermis but did not involve deeper layers. A distance between the wounds of at least $5 \mathrm{~cm}$ was respected. The procedure caused only minor pain, so no anesthetic was necessary.

\section{Study Products, Dosage, and Administration}

The test product, BepanGel wound care hydrogel (Flamozil, Zoralan Wound), is classified as a IIb Medical Device (with CE mark) and meets requirements of the European Council Directive 93/42 EEC. BepanGel is composed of acidic colloidal carbomer, carnosine, water, and preservative (sodium benzoate, potassium sorbate) and is manufactured by Oystershell Laboratories (Merelbeke, Belgium). BepanGel is available in a plastic tube of $20 \mathrm{~g}$ and $50 \mathrm{~g}$ sizes. In this clinical investigation, $50 \mathrm{~g}$ plastic tubes were used. The test fields were randomly assigned in all subjects with the hydrogel kept at room temperature before application. It was topically applied (approximately $0.2 \mathrm{~g}$ BepanGel for a single dosage) for a 12-day treatment period, covering 11 treatments (approximately $2.2 \mathrm{~g}$ BepanGel in total). The hydrogel was carefully distributed over the test field, but not rubbed in. The other test field remained untreated. Both test fields were covered with standard semi-occlusive wound plasters (Hansaplast Sensitive wound plaster) for protection. This routine was repeated daily over the test period by an unblinded study nurse at bioskin RCD in Hamburg, Germany.

\section{Wound Healing Efficacy, Cooling, Tolerability, and Cosmetic Appearance Evaluation}

Wound healing efficacy assessments as well as the clinical assessment of tolerability and evaluation of signs of infection were performed by a blinded investigator on days $2,6,8,10$, and 12 , while cooling property (day 1 ), product performance, and product traits (day 12) were assessed by the unblinded subjects. Global assessment of local tolerability and the cosmetic outcome were evaluated both by the blinded investigator and the unblinded subjects throughout the investigation (days 2, 6, 8, 10, and 12) and during follow-up at the end of the investigation (day 31 ), respectively.

\section{Assessment of Wound Healing}

Wound healing efficacy of both test sites was evaluated by a blinded investigator by scoring using a 6-point re-epithelialization scale $(0=0 \%$ healing; $1=1-25 \%$ re-epithelialization; $2=26-50 \%$ re-epithelialization; $3=51-75 \%$ reepithelialization; $4=$ over $75 \%$ but not complete re-epithelialization; $5=100 \%$ complete healing). A global assessment of wound healing was performed by the blinded investigator on the basis of a 5-point scale $(0=$ very good; 1 = good; 2 = acceptable; 3 = poor; 4 = very poor). Also, the total time until complete healing for both test fields was evaluated, as determined by the first day after wound induction with a re-epithelialization score of 5 . Additionally, the presence of crusts was assessed (yes/no) for the two test fields.

\section{Assessment of Cooling Effect}

On day 1 of the clinical investigation, the cooling effect in the two test fields was assessed by the unblinded subjects using a visual 
analogue scale (VAS) of $100 \mathrm{~mm}$ length, ranging from 0 (no cooling) to 100 (extremely cooling), $20 \pm 5 \mathrm{~s}$ after application of BepanGel, but prior to application of the protective wound plaster. The assessment was performed for the untreated test field as well. A VAS is commonly used to measure pain, itching, and other subjective responses and has been proven to have a high reliability and concurrent validity $[12,13]$. Specifically, VAS have also been used in assessing thermal perception for decades [14, 15].

\section{Assessment of Tolerability}

The clinical tolerability was assessed by a blinded investigator by scoring using a 5-point erythema scale on healthy skin in close proximity to the two test fields $(0=$ no reaction; $1=$ slight uniform or spotty erythema or slight diffuse, partial or follicular erythema; $2=$ clear, sharply demarcated erythema; $3=$ severe erythema with infiltrate; $4=$ severe erythema with infiltrate and/or epidermal defect [blisters, blebs, erosions]). Additionally, the tolerability was globally assessed by the blinded investigator and the unblinded subject by means of a 5 -point scale $(0=$ very good; $1=$ good; $2=$ acceptable; $3=$ poor; $4=$ very poor).

Furthermore, signs of infection were assessed for both test fields by the blinded investigator by answering a closed question (yes/no), considering the following parameters: erythema, pain, malodor, delayed wound healing, excessive exudate, and heat.

\section{Assessment of Cosmetic Outcome and Acceptance}

The cosmetic outcome/acceptance was assessed on day 31 by the blinded investigator and the unblinded subject by means of a VAS of $100 \mathrm{~mm}$ length, ranging from 0 (poor) to 100 (excellent) for each of the test fields.

\section{Product Performance}

Twelve questions related to product performance and product traits were filled out by all unblinded subjects using a 7-tiered rating scale ( 1 = strongly agree; $2=$ moderately agree; $3=$ slightly agree; $4=$ neither agree nor disagree; $5=$ slightly disagree; $6=$ moderately disagree; $7=$ strongly disagree) on day 12 of the investigation.

\section{Safety Evaluation}

The abrasive wound test model did not pose a relevant risk for the subjects, as demonstrated by past experiences with the induction of small superficial wounds that usually heal with no consequences. Small scars may occur as a result of injury to the dermis [16]. For the test product, all required quality, safety, and efficacy issues are addressed and evaluated on the basis of the documentation essential to fulfil the requirements of the European Council Directive 93/42 EEC. According to the Risk Management Procedure for the medical device in compliance with ISO 14971, no unacceptable risks were identified.

Serious adverse events (SAEs) were reported within $24 \mathrm{~h}$ from the acknowledgement to the sponsor. Spontaneously noted complaints were recorded as AEs with duration, intensity, and probability of a correlation with the test product.

\section{Data Analysis and Statistics}

The statistical evaluation was performed at bioskin $\mathrm{GmbH}$ using the software program SAS (Statistical Analysis System, SAS Inc., Cary, NC) and data were analyzed by a statistician according to the International Conference on Harmonization (ICH) E9 Note for Guidance on Statistical Principles for Clinical Trials [17].

For the re-epithelialization score and global assessment of wound healing efficacy scores, the area under the curve (AUC) was calculated using the trapezoid formula and compared by means of a paired $t$ test. For the assessment of cooling properties by subject on VAS (day 1 ) and global tolerability (days 2, 6, 8, 10, and 12), mean scores over day 1 or all visits, respectively, were presented descriptively by treatment. 


\section{RESULTS}

\section{Baseline Data Clinical Trial}

The clinical investigation was carried out between January and February 2020. In total, 40 healthy volunteers were screened for eligibility and 33 subjects were included. Seven subjects were not randomized: six subjects were allocated as backup subjects in case of need for replacements and one subject had schedule problems. The two induced wounds on each subject were randomly treated with one of the two treatment regimens (BepanGel wound care hydrogel covered with standard semi-occlusive wound plaster or only covered with standard semi-occlusive wound plaster without hydrogel) for 11 consecutive days. In total, 32 subjects completed the study according to protocol. In one subject, two SAEs unrelated to the hydrogel or the study procedures were reported and finally led to premature subject discontinuation. The last visit of this subject was on day 12; hence, the subject was included in the analyses, but missed the follow-up visit. A CONSORT flowchart providing an overview of the clinical

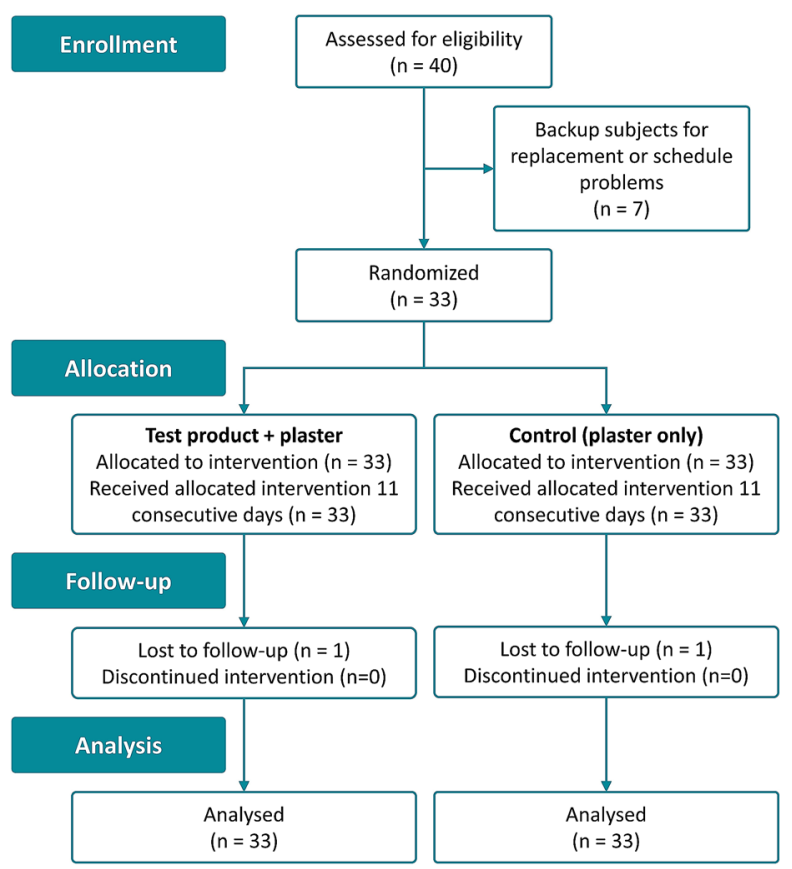

Fig. 1 CONSORT flowchart (within-person trials) investigation process is shown in Fig. 1. Subject demographics and baseline characteristics are summarized in Table 1.

\section{Wound Healing Efficacy}

Already starting on day 6 after wound induction, the hydrogel-treated test fields showed a significantly faster onset of wound closure as demonstrated by wound re-epithelialization. As such, re-epithelialization scores were consistently higher in the hydrogel-treated test fields at all time points in comparison with the test fields treated with a plaster alone, as demonstrated in Table 2 and Fig. 2 . At the end of the investigation on day 12, the BepanGel-treated test field was completely healed in nearly all subjects (97.0\%). In the test fields treated with a plaster alone, only six subjects demonstrated complete healing (18.2\%). Increased re-epithelialization after treatment with the hydrogel was also reflected by a significantly larger mean $\mathrm{AUC}_{\text {days 2-12 }}$ for the hydrogel-treated test fields in comparison with test fields treated with a plaster alone as shown in Table 2. The higher reepithelialization rate in the BepanGel-treated test fields was accompanied by a reduced time to complete healing in comparison with the test fields treated with the plaster alone as illustrated in Fig. 3 (18 BepanGel-treated test fields were completely healed by day 10 , and a further 14

Table 1 Subject demographics and baseline characteristics

\begin{tabular}{ll}
\hline Number of subjects & 33 \\
Age (mean \pm SD) & $55.6 \pm 10.8$ (years) \\
Sex & $18(55 \%)$ \\
Male & $15(45 \%)$ \\
Female & \\
Skin type (Fitzpatrick) & $33(100 \%)$ \\
I-III & $0(0 \%)$ \\
IV-VI & \\
Race & $33(100 \%)$ \\
Caucasian
\end{tabular}


Table 2 Mean re-epithelialization values of test fields treated with BepanGel and plaster or plaster alone

\begin{tabular}{|c|c|c|c|c|c|c|}
\hline Re-epithelialization & $\begin{array}{l}\text { Day } 2 \\
(\operatorname{man} \pm S D)\end{array}$ & $\begin{array}{l}\text { Day } 6 \\
(\text { mean } \pm S D)\end{array}$ & $\begin{array}{l}\text { Day } 8 \\
(\text { mean } \pm S D)\end{array}$ & $\begin{array}{l}\text { Day } 10 \\
(\text { mean } \pm S D)\end{array}$ & $\begin{array}{l}\text { Day } 12 \\
(\text { mean } \pm S D)\end{array}$ & $\mathrm{AUC}_{\text {days 2-12 }}$ \\
\hline $\begin{array}{l}\text { BepanGel + plaster } \\
\quad(n=33)\end{array}$ & $0.0 \pm 0.0$ & $1.6 \pm 0.6$ & $2.8 \pm 0.8$ & $4.4 \pm 0.7$ & $5.0 \pm 0.2$ & $24.2 \pm 3.5^{* * *}$ \\
\hline Plaster $(n=33)$ & $0.0 \pm 0.0$ & $0.9 \pm 0.3$ & $1.6 \pm 0.7$ & $3.0 \pm 1.1$ & $4.0 \pm 0.7$ & $16.0 \pm 4.4$ \\
\hline
\end{tabular}

$A U C$ area under the curve

${ }^{* * *} p<0.0001$

by day 12 . Over $75 \%$ but not complete re-epithelialization was noted in the remaining one subject. In comparison, when treated with a plaster alone, four test fields were healed by days 10 and a further two by day $12 ; 27$ test fields were partially healed by day 12 ).

In addition, the investigator assessed the global wound healing as predominantly "very good" at each assessment point in the hydrogeltreated test fields. In the test fields treated with the plaster alone, the global wound healing was assessed as "very good" in fewer subjects at each assessment point. This resulted in a significantly smaller $\mathrm{AUC}_{\text {days 2-12 }}$ in the hydrogel-treated test fields $(0.3 \pm 1.1)$ in comparison to the test fields treated with the plaster alone $(3.3 \pm 2.7$, $p<0.0001$ ), again confirming better healing efficacy of the wounds treated with BepanGel. Crust formation was observed in only 2 of 33 subjects $(6.1 \%)$ in both test fields.

\section{Cooling Properties}

Immediately after the first administration of the hydrogel, about half of the subjects (48.5\%) assessed a slight cooling effect for BepanGel on the VAS (mean $9.7 \pm 20.5$ ). Fewer subjects (39.4\%) assessed a cooling effect in the untreated test field (mean $4.5 \pm 11.4$ ), although this did not achieve statistical significance $(p=0.1441)$ in comparison with the hydrogeltreated test field.

At day 12 of the investigation, two-thirds of the subjects strongly, moderately, or slightly agreed that BepanGel provides an immediate cooling effect when answering the questionnaire $(p=0.0555)$.

\section{Safety Assessment}

During the investigation, no wound gel-related (S)AEs were reported. One subject reported two SAEs (rupture quadriceps tendon right side and rupture quadriceps tendon left side on day 11 and day 27, respectively), which led to premature discontinuation of the investigation. Both SAEs were recovering/resolving at the end of this clinical investigation. No other serious or non-serious AEs or any device deficiencies were reported. In addition, no signs of infection were reported by the investigator in any of the two test fields over the entire investigation period.

\section{Tolerability Assessment}

Generally, BepanGel wound care hydrogel demonstrated an excellent local tolerability when applied to standardized, artificially induced abrasive wounds. The clinical assessment by the investigator showed that there was no local reaction on healthy skin in close proximity to the two test fields at day 2 in approximately two-thirds of the subjects after treatment with BepanGel and plaster alone. In the remaining subjects, a slight erythema was noted for both test fields (11 BepanGel-treated test fields and 9 test fields treated with plaster alone demonstrated slight erythema). At the other time points, no reaction was seen in either of the test fields except for one or two subjects presenting with slight erythema. There was no significant difference in general clinical tolerability between the hydrogel and plaster (mean sum $0.5 \pm 0.7$ ) or plaster alone (mean sum $0.4 \pm 0.5$ ) over the 12 investigation days. 


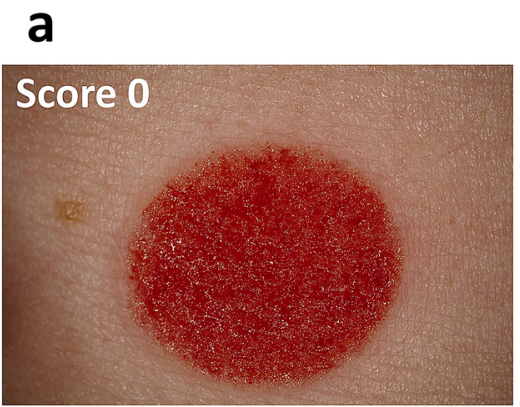

0\% healing

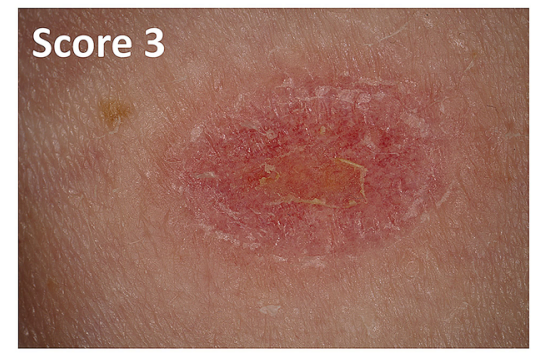

$51-75 \%$ re-epithelialization

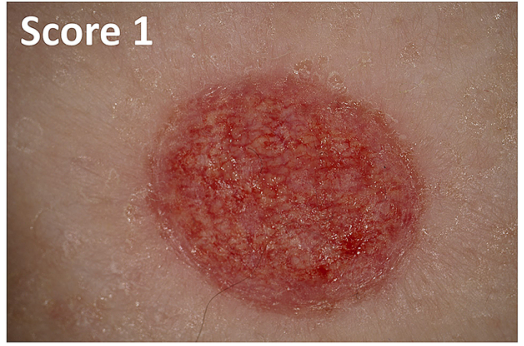

$1-25 \%$ re-epithelialization

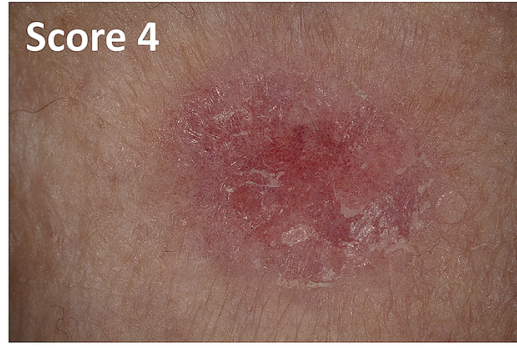

$>75 \%$ but not complete reepithelialization

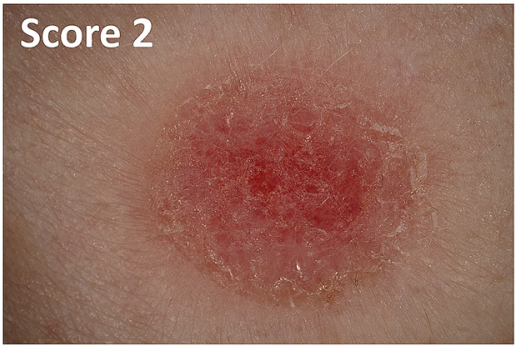

$26-50 \%$ re-epithelialization

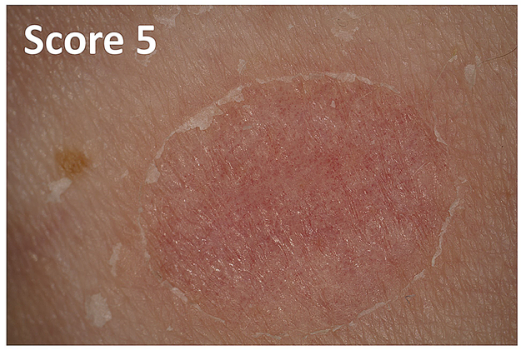

$100 \%$ complete healing

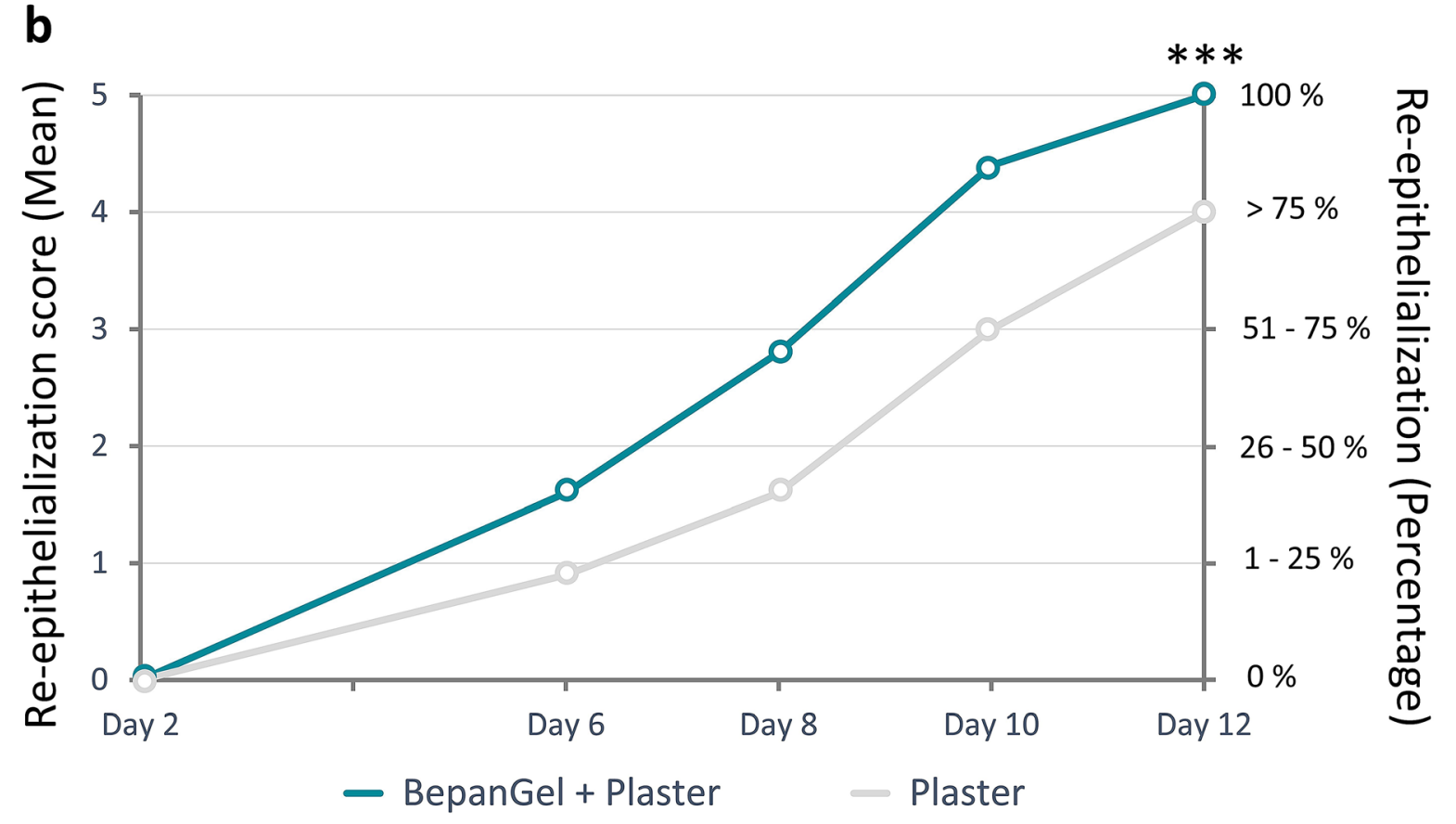


4Fig. 2 Example of wound healing assessment of induced abrasive wounds by means of a 6-point-re-epithelialization scale. a Score $0=0 \%$ healing; score $1=1-25 \%$ reepithelialization; score $2=26-50 \%$ re-epithelialization; score $3=51-75 \%$ re-epithelialization; score $4=$ over $75 \%$ but not complete re-epithelialization; score $5=100 \%$ complete healing. b Visual illustration of re-epithelialization of abrasive wound test fields treated with BepanGel in comparison with test fields treated with a plaster alone over a treatment period of 12 days. ${ }^{* * *} p<0.0001$

The global tolerability rated by the subjects for both test fields was overall "very good" and "good" over all time points for both test fields with scores increasing over time. No statistical difference was observed between the two test fields, although slightly better results were obtained with BepanGel (mean sum $2.1 \pm 2.5$ for the BepanGel-treated test field and mean sum $2.7 \pm 3.0$ for the test field treated with a plaster alone) over the total investigation period. As for the blinded investigator, global tolerability was scored predominantly "very good" for both test fields during the study. The global tolerability was statistically similar between the test product (mean sum $0.2 \pm 0.6$ ) and the test field treated with the plaster alone (mean sum $0.2 \pm 0.5)$. An overview of the tolerability results is provided in Fig. 4 and further details can be found in Table S1 in the Electronic Supplementary Material.

In addition, no signs of infection were observed throughout the total investigational period of 31 days.

\section{Cosmetic Appearance and Acceptance}

The cosmetic appearance of the test fields was evaluated both by the subjects and the blinded investigator on day 31 of the clinical investigation through VAS assessments. The subjects rated the cosmetic appearance of the hydrogeltreated test field on average $81.9 \pm 18.9$ in comparison with $71.5 \pm 22.8$ for the test field treated with a plaster alone $(p=0.0078)$. These results were similar to the rating of the blinded investigator, who rated the BepanGel-treated test field on average $83.6 \pm 17.6$ and the plastertreated test field $72.3 \pm 21.2(p=0.0005)$.

\section{Product Performance and Product Traits}

Lastly, the effect of BepanGel on the test fields was evaluated by the subjects on performance and traits through a questionnaire. In general, $87.9 \%$ of the subjects were of the opinion that the gel has a pleasant texture and $51.5 \%$ moderately to strongly agreed that BepanGel provides immediate pain relief. As such, at least

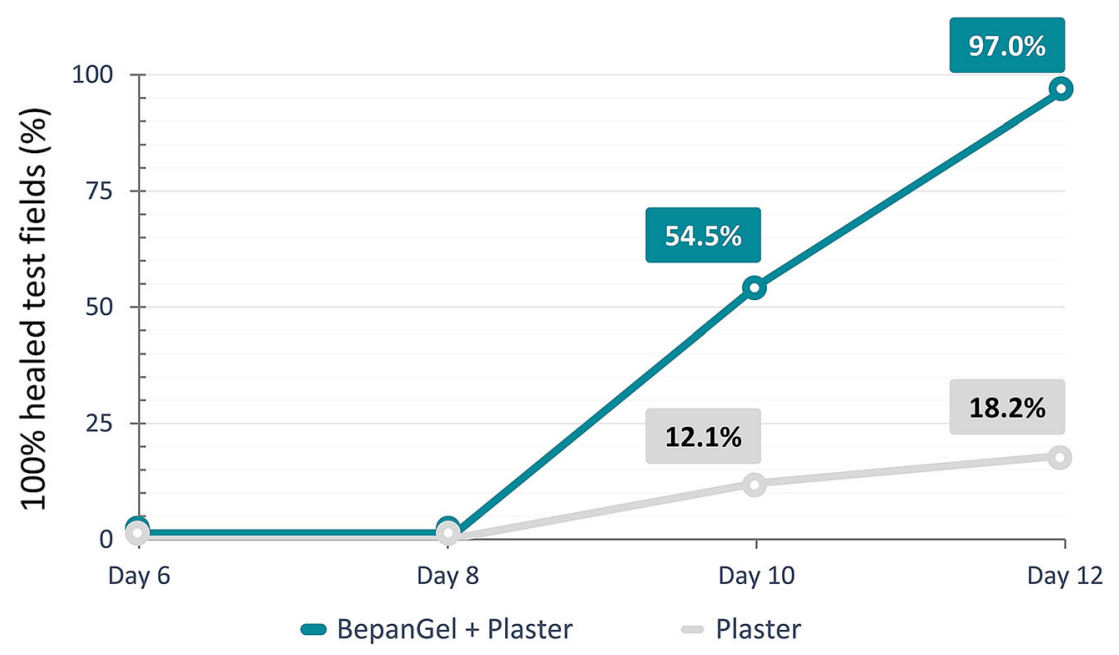

Fig. 3 Graph demonstrating the time to complete healing of induced abrasive wounds after BepanGel treatment and treatment with a plaster alone 

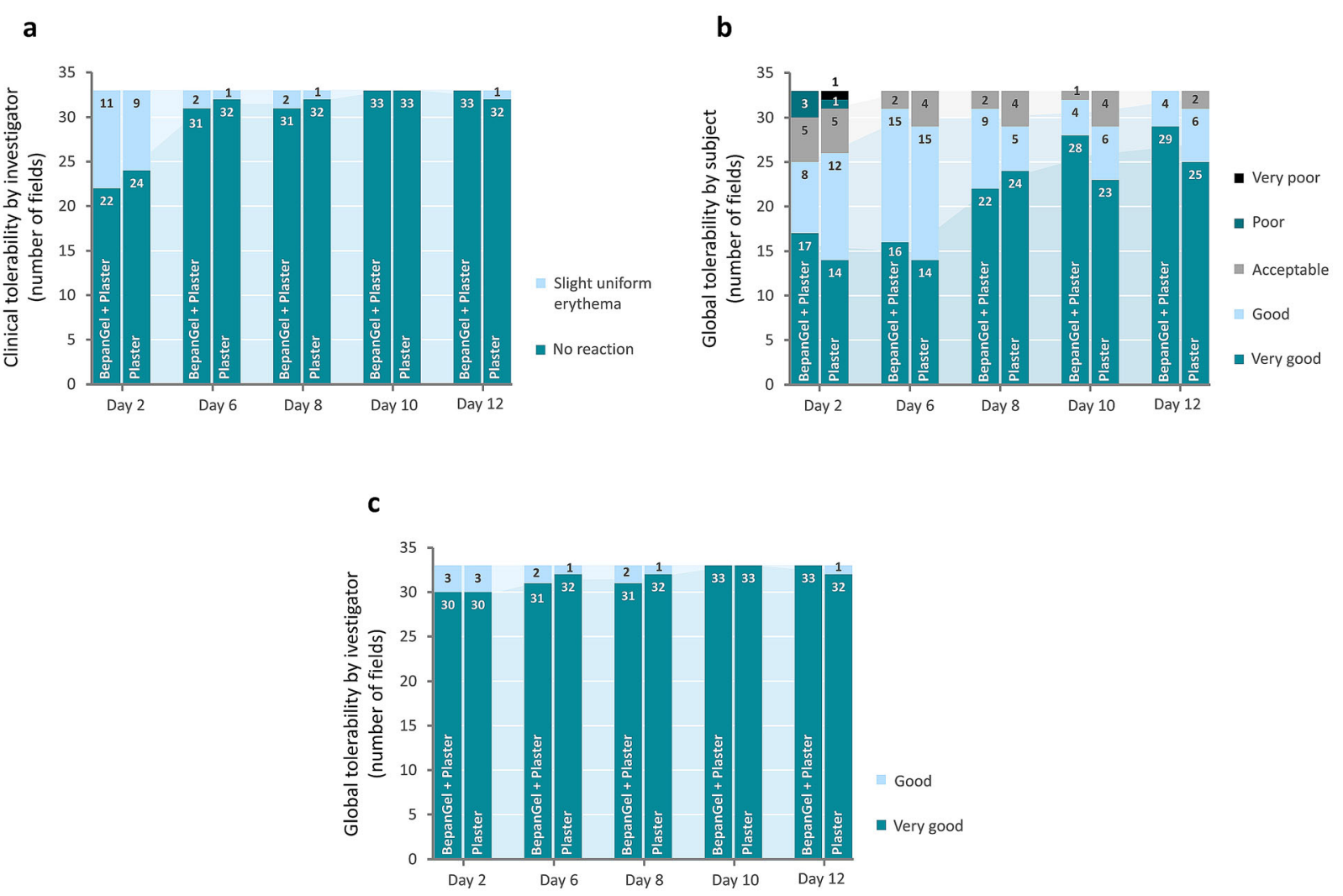

Fig. 4 Tolerability assessments by the blinded investigator and the unblinded subjects. a Clinical tolerability evaluation of the BepanGel-treated test field and the plastertreated test fields by the investigator. $\mathbf{b}$ Global tolerability evaluation by the subjects of the BepanGel-treated test field in combination with a plaster in comparison with a

three-quarters of the study population acknowledged a reduced pain sensation during and after application with the hydrogel as well as when the bandage was changed. Finally, $90.91 \%$ of the subjects agreed that the hydrogel speeds up the healing process. Results of the questionnaire, limited to the questions relevant for the current investigation, are provided in Table 3.

\section{DISCUSSION}

Hydrogels are three-dimensional polymer networks capable of holding large amounts of water which makes them very appealing for all kinds of biological applications [18]. Their first plaster alone. c Global tolerability evaluation by the investigator of the BepanGel-treated test fields in combination with a plaster in comparison with a plaster alone

clinical application was as soft contact lenses in 1960 after these water-based polymers were discovered in 1936 by DuPont (du Pont de Nemours 1936) [19]. Since then, hydrogel technologies have been widely used for several purposes including hygiene products, drug delivery systems, sealing, pharmaceuticals, biomedical applications, tissue engineering, regenerative medicines, and wound dressings [20]. As a result of their structure, hydrogels possess many desired characteristics to effectively treat wounds, including cleansing of dry wounds by providing a moist environment, providing permeability towards metabolites, and their ability to cool the surface of the wound leading to an immediate relief of pain $[6,21,22]$. In addition, the hydrogel forms a 
Table 3 BepanGel product performance and product traits questionnaire

\begin{tabular}{llllr}
\hline Question & $\boldsymbol{N}$ & $\boldsymbol{N}$ agreement & \% agreement & $\boldsymbol{p}$ value $^{\mathbf{a}}$ \\
\hline The gel has a pleasant texture & 33 & 32 & 96.97 & $<0.0001$ \\
The gel provides immediate cooling effect & 33 & 22 & 66.67 & 0.0555 \\
The gel provides immediate pain relief & 33 & 21 & 63.64 & 0.1172 \\
The gel reduces sensation of pain during application & 33 & 26 & 78.79 & 0.0009 \\
The gel reduces sensation of pain after application & 33 & 27 & 81.82 & 0.0003 \\
The gel speeds up healing time & 33 & 30 & 90.91 & $<0.0001$ \\
No pain while changing bandage & 33 & 31 & 93.94 & $<0.0001$ \\
\hline
\end{tabular}

The questions provided in the table are a selection of the complete questionnaire. The questions and respective answers presumed most relevant to the investigation are provided

${ }^{a} p$ value from a two-sided binomial test for null hypothesis proportion $p 0=0.5$ to test if the agreement level differs from $50 \%$ with (nominal) significance level of 5\%

physical barrier preventing contamination and potential infection of the wound.

BepanGel (Flamozil, Zoralan Wound) is a wound care hydrogel that promotes wound healing by regulating the microenvironment of the wound and serving as a barrier between the healing wound and external factors. Presence of the carbomer polymer ensures optimal hydration-and subsequently optimal conditions for healing-of wounds at all times by releasing moisture into the wound bed of dry wounds and taking up moisture (exudates) of wet wounds. In this way, BepanGel serves as an ideal treatment for several types of wounds including superficial burns, grazed skin, abrasions and cuts, surgical wounds, bedsores, and open leg sores. In addition, the low $\mathrm{pH}$ of the hydrogel prevents microbial growth in a natural way.

The current investigation clearly substantiates the beneficial effects of BepanGel on healing of everyday superficial wounds in a clinical setting in comparison with the standard treatment consisting of wound plasters. First and foremost, complete wound healing as assessed by re-epithelialization of the wounds was noted in $97.0 \%$ of all hydrogel-treated test fields (of which $54.5 \%$ of the test fields were already completely healed by day 10). This was only the case for $18.2 \%$ of the test fields treated with standard plasters alone at the end of the investigation. These results were confirmed by a larger $\mathrm{AUC}_{\text {days 2-12 }}(p<0.0001)$ regarding reepithelialization for the test field treated with the hydrogel and a significantly better scoring of global wound healing by the blinded investigator. An earlier closure of wounds is of clinical relevance as it minimizes the risk of secondary infections.

In general, it should be considered that both test fields were covered with a standard semiocclusive wound dressing which mostly alone results in a better wound healing outcome than under uncovered, dry conditions. Therefore, the difference in wound healing of the covered BepanGel-treated test field to a fully unprotected, untreated test field might be expected to be even more pronounced.

Despite previously proven cooling properties of hydrogel due to their high water content, no statistical significance was reached in the current clinical evaluation in regard to cooling properties of the hydrogel. It should be noted, however, that at the end of the study about twothirds of the subjects agreed that BepanGel provides an immediate cooling effect $(p=0.0555)$. The lack of remarkable cooling experience is probably due to the small size of the wounds, making it nearly impossible to become aware of temperature differences. One way to test this hypothesis is to treat a larger test 
wound surface area with the hydrogel; however, that would not have been ethically justifiable.

During follow-up of the investigation on day 31 , both the unblinded study population and the blinded investigator rated the cosmetic appearance of the test field treated with hydrogel better than treatment with the plaster alone ( $p=0.0005$ and $p=0.0078$, respectively). These findings are consistent with the beneficial wound healing properties of the hydrogel speeding up the healing process by promoting wound epithelialization and wound closure, and subsequently reduce scarring.

It is important to note that the hydrogel's performance was very positively rated by the study population-and potential end users of the marketed test product-with regard to texture and provision of pain relief during and after application, as well as when changing the bandage.

Lastly, BepanGel demonstrated excellent local tolerability when applied to standardized, artificially induced abrasive wounds, while no AEs associated with the use of the hydrogel were reported over those already known and described.

A limitation of the current study is a comparison of BepanGel-treated wounds with a test field without any covering of the wound. The reason for this decision is threefold: (1) the standard of care of small, everyday epidermal wounds currently consists of a semi-occlusive plaster and hence serves as a relevant control, (2) if BepanGel-treated test fields remain uncovered, this will most likely result in removal of the wound hydrogel by scrubbing against clothes or obstacles, leaving the wounds with an insufficient amount of BepanGel, and (3) covering of the BepanGel-treated test fields limits the evaporation of water from the hydrogel, ensuring optimal treatment conditions. Another limitation is the small surface (1.2 cm diameter) of the induced wounds which might explain the absence of a significant cooling effect and difference in cosmetic appearance of the wounds. However, a larger test field area would not be in line with ethical considerations.

\section{CONCLUSION}

The results of this clinical investigation clearly demonstrate that there is a relevant benefit of using BepanGel wound care hydrogel for the daily treatment of small epithelial wounds over plaster alone. The hydrogel is easy in use and improves wound healing efficacy by enhanced re-epithelialization, leading to a better cosmetic outcome while providing a slight cooling effect upon application. BepanGel is well tolerated by all study subjects to the same extent as standard treatment with a plaster alone.

\section{ACKNOWLEDGEMENTS}

Funding. Oystershell Laboratories sponsored this study and is also the manufacturer of the investigational product. Study funding and the journal's rapid service fee were funded by Oystershell Laboratories and Bayer Consumer Care AG. The sponsor was involved in the study planning as well as in the decision to publish, but was not involved in data collection, data analysis, and data interpretation. All authors had full access to all data in this study and take complete responsibility for the integrity of the data and accuracy of the data analysis.

Authorship. All named authors meet the International Committee of Medical Journal Editors (ICMJE) criteria for authorship for this article, take responsibility for the integrity of the work as a whole, and have given their approval for this version to be published.

Medical Writing and Other Assistance. Oystershell Laboratories engaged bioskin $\mathrm{GmbH}$ to independently design and perform the clinical investigation. Writing of the manuscript was performed by an independent, external consultant, Leni Vandekerckhove (email: lenivandekerckhove@hotmail.com). We thank all subjects for participating in the clinical trial.

Disclosures. Lei Zang, Raffaella de Salvo, and Sonja Trapp are employees of Bayer Consumer Care AG, which partially funded this 
study. Walter Wigger-Alberti and Ragna Williams are employees of bioskin $\mathrm{GmbH}$, which performed the study. Lucie Delcour, Bart Rossel, and Maarten Huisman are employees of Oystershell Laboratories, which is the developer of the test product and partially funded this study.

Compliance with Ethics Guidelines. The clinical investigation was conducted in accordance with local law, the Declaration of Helsinki 1964 and its later amendments, and ISO 14155:2011. Written informed consent was obtained for all included subjects. The study was approved by the ethics committee of the medical association in Hamburg (Ethik-Kommission der Ärztekammer Hamburg, Weidestraße 122b, 22083 Hamburg, Germany).

Data Availability. The datasets generated during and/or analyzed during the current study are available from the corresponding author on reasonable request.

Open Access. This article is licensed under a Creative Commons Attribution-NonCommercial 4.0 International License, which permits any non-commercial use, sharing, adaptation, distribution and reproduction in any medium or format, as long as you give appropriate credit to the original author(s) and the source, provide a link to the Creative Commons licence, and indicate if changes were made. The images or other third party material in this article are included in the article's Creative Commons licence, unless indicated otherwise in a credit line to the material. If material is not included in the article's Creative Commons licence and your intended use is not permitted by statutory regulation or exceeds the permitted use, you will need to obtain permission directly from the copyright holder. To view a copy of this licence, visit http://creativecommons.org/licenses/by$\mathrm{nc} / 4.0 /$.

\section{REFERENCES}

1. Enoch S, Price P. Cellular, molecular and biochemical differences in the pathophysiology of healing between acute wounds, chronic wounds and wounds in the aged. World Wide Wounds 2004. http://www.worldwidewounds.com/2004/ august/Enoch/Pathophysiology-Of-Healing.html. Accessed 30 July 2020.

2. Atiyeh BS, Ioannovich J, Al-Amm CA, El-Musa KA. Management of acute and chronic open wounds: the importance of moist environment on optimal wound healing. Curr Pharmaceut Biotech. 2002;3: 179-95.

3. Korting HC, Schoellmann C, White RJ. Management of minor acute cutaneous wounds: importance of wound healing in a moist environment. J Eur Acad Dermatol Venereol. 2011;25(2):130-7.

4. Kuhlmann M, Wigger-Alberti W, Mackensen VY, et al. Wound healing characteristics of a novel wound healing ointment in an abrasive wound model: a randomised, intra-individual clinical investigation. Wound Med. 2019;24(1):24-32.

5. Trudgian J. Investigating the use of aquaform hydrogel in wound management. $\mathrm{Br} \mathrm{J}$ Nurs. 2000;9(14):943-8.

6. da Silva LP, Reis RL, Correlo VM, Marques AP. Hydrogel-based strategies to advance therapies for chronic skin wounds. Annu Rev Biomed Eng. 2019;21:145-69.

7. Grinstaff MW. Designing hydrogel adhesives for corneal wound repair. Biomaterials. 2007;28(35): 5205-14.

8. Stubbe B, Mignon A, Declercq H, Van Vlierberghe S, Dubruel P. Development of gelatin-alginate hydrogels for burn wound treatment. Macromol Biosci. 2019;19(8):e1900123.

9. Edwards J. Hydrogels and their potential uses in burn wound management. Br J Nurs. 2010;19(11): S12 S14-6.

10. Wigger-Alberti W, Kuhlmann M, Ekanayake S, et al. Using a novel wound model to investigate the healing properties of products for superficial wounds. J Wound Care. 2009;18(3):123-31.

11. US Food and Drug Administration. Assessing the irritation and sensitization potential of transdermal and topical delivery systems for ANDAs guidance for industry. Draft guidance (2018). Docket Number: FDA-2018-D-3546. https://www.fda.gov/ media/117569/download. Accessed 30 July 2020.

12. Phan NQ, Blome C, Fritz F, et al. Assessment of pruritus intensity: prospective study on validity and reliability of the visual analogue scale, numerical rating scale and verbal rating scale in 471 patients 
with chronic pruritus. Acta Derm Venereol. 2012;92:502-50.

13. Hjermstad MJ, Fayers PM, Haugen DF, et al. Studies comparing numerical rating scales, verbal rating scales, and visual analogue scales for assessment of pain intensity in adults: a systematic literature review. J Pain Symptom Manag. 2011;41(6): 1073-93.

14. Leon GR, Koscheyev VS, Stone EA. Visual analog scales for assessment of thermal perception in different environments. Aviat Space Environ Med. 2008;79(8):784-6.

15. Gong P, Wang N, Guan L, Lai W. Two randomized studies to evaluate the cooling sensation, consumer liking, and tolerability of a skin disinfectant spray. J Cosmet Sci. 2017;68(3):205-17.

16. Bolognia JL, Jorizzo JL, Rapini RP. Dermatology second edition. Marylad Heights: Mosby Elsevier; 2008. p. 2147-58.
17. International Conference on Harmonization (ICH). Statistical principles for clinical trials. Fed Reg. 1998;63:49583.

18. Peppas AN, Hilt JZ, Khademhosseini A, Langer R. Hydrogels in biology and medicine: from molecular principles to bionanotechnology. Adv Mater. 2006;18:1345-60.

19. Wichterle O, Lím D. Hydrophilic gels for biological use. Nature. 1960;1(85):117-8.

20. Ahmed EM. Hydrogel: preparation, characterization, and applications: a review. J Adv Res. 2015;6(2):105-21.

21. Boateng JS, Matthews KH, Stevens HN, Eccleston GM. Wound healing dressings and drug delivery systems: a review. J Pharm Sci. 2008;97:2892-923.

22. Harrison IP, Spada F. Hydrogels for atopic dermatitis and wound management: a superior drug delivery vehicle. Pharmaceutics. 2018;10(2):71. 